\title{
Hankel Determinant for a Subclass of Alpha Convex Functions
}

\author{
Gagandeep Singh and Gurcharanjit Singh
}

\begin{abstract}
In the present investigation, the upper bound of second Hankel determinant $\left|a_{2} a_{4}-a_{3}^{2}\right|$ for functions belonging to the subclass $M(\alpha ; A, B)$ of analytic functions is studied. Results presented in this paper would extend the corresponding results of various authors.
\end{abstract}

Keywords--- Analytic Functions, Starlike Functions, Convex Functions, Alpha Convex Functions, Subordination, Schwarz Function, Second Hankel Determinant

\section{INTRODUCTION}

LET $A$ be the class of analytic functions of the form

$$
f(z)=z+\sum_{k=2}^{\infty} a_{k} z^{k}
$$

In the unit $\operatorname{disc} E=\{z:|z|<1\}$.

By $S$ we denote the class of functions $f(z) \in A$ and univalent in $E$.

Let $U$ be the class of Schwarzian functions

$$
w(z)=\sum_{k=1}^{\infty} d_{k} z^{k}
$$

Which are analytic in the unit disc $E=\{z:|z|<1\}$ and satisfying the conditions $w(0)=0$ and $|w(z)|<1$.

Let $f$ and $g$ be two analytic functions in $E$. Then $f$ is said to be subordinate to $g$ (symbolically $f \prec g$ ) if there exists a Schwarz function $w(z) \in U$, such that $f(z)=g(w(z))$.

In 1976, Noonan and Thomas [9] stated the qth Hankel determinant of $f(z)$ for $q \geq 1$ and $n \geq 1$ as

$$
H_{q}(n)=\left|\begin{array}{cccc}
a_{n} & a_{n+1} & \ldots & a_{n+q-1} \\
a_{n+1} & \ldots & \ldots & \ldots \\
\ldots & \ldots & \ldots & \ldots \\
a_{n+q-1} & \ldots & \ldots & a_{n+2 q-2}
\end{array}\right| .
$$

Gagandeep Singh, Department of Mathematics, M.S.K. Girls College, Bharowal(Tarn-Taran), Punjab, India. E-mail: kamboj.gagandeep@yahoo.in Gurcharanjit Singh, Department of Mathematics, Guru Nanak Dev University College, Chungh(Tarn-Taran), Punjab, India. E-mail: dhillongs82@yahoo.com
The Hankel determinant plays an important role in the study of singularities; for instance, see[2]. This is also important in the study of power series with integral coefficients, see [2] and Cantor[1]. Hankel determinants are useful in showing that a function of bounded characterstic in the unit disc i.e. a function which is a ratio of two bounded analytic functions with its Laurent series around the origin having integral coefficients, is rational. It is well known that the Fekete-Szegö functional

$$
\left|a_{3}-a_{2}^{2}\right|=H_{2}(1)
$$

This functional is further generalized as $\left|a_{3}-\mu a_{2}^{2}\right|$ for $\mu$ (real as well as complex). It is a very great combination of the two coefficients which describes the area problems posted earlier by Gron wall in 1914-15. Moreover, we also know that the functional $\left|a_{2} a_{4}-a_{3}^{2}\right|$ is equivalent to $H_{2}(2)$. The functional $\left|a_{2} a_{4}-a_{3}^{2}\right|$ has been studied by many authors see ([4-6],[10],[14-16]). Janteng et al. [4] have considered the functional $\left|a_{2} a_{4}-a_{3}^{2}\right|$ and found a sharp bound for the function $f$ in the subclass $R$ of $S$, consisting of functions whose derivative has a positive real part studied by MacGregor[9]. In their work, they have shown that if $f \in R$, then $\left|a_{2} a_{4}-a_{3}^{2}\right| \leq \frac{4}{9}$. The same authors [5] also obtained the second Hankel determinant and sharp upper bounds for the familier subclasses namely, starlike and convex functions denoted by $S^{*}$ and $K$ of $S$ and have shown that $\left|a_{2} a_{4}-a_{3}^{2}\right| \leq 1$ and $\left|a_{2} a_{4}-a_{3}^{2}\right| \leq \frac{1}{8}$ respectively.

Motivated by the above mentioned results obtained by different authors in this direction, in this paper, we introduce certain subclass of analytic functions and obtain an upper bound to the functional $\left|a_{2} a_{4}-a_{3}^{2}\right|$ for the function $f$ belonging to this class, defined as follows:

$M(\alpha ; A, B)$ denote the subclass of functions $f(z) \in A$ and satisfying the condition 


$$
(1-\alpha) \frac{f^{f^{\prime}(z)}}{f(z)}+\alpha \frac{\left(f^{\prime}(z)\right)^{\prime}}{f^{\prime}(z)} \prec \frac{1+A z}{1+B z},-1 \leq B<A<1,0 \leq \alpha \leq 1, z \in E
$$

The class $M(\alpha ; A, B)$ is the subclass of alpha-convex functions. The following observations are obvious:

- $M(\alpha, 1,-1) \equiv M(\alpha)$, the class introduced by Mocanu[11].

- $\quad M(0,1,-1) \equiv S^{*}$, the class of starlike functions.

- $M(1 ; 1,-1) \equiv K$, the class of convex functions.

- $M(0 ; A, B) \equiv S^{*}(A, B)$, the subclass of starlike functions studied by Goel and Mehrok [3].

- $M(1 ; A, B) \equiv K(A, B)$, the subclass of convex functions studied by Goel and Mehrok [3].

Results due to Janteng et al.[5] and Singh and Singh [16] follows as special cases from the results of this paper.

\section{PReliminary Results}

Let $P$ be the family of all functions $p$ analytic in $E$ for which $\operatorname{Re}(p(z))>0$ and

$$
p(z)=1+p_{1} z+p_{2} z^{2}+\ldots
$$

for $Z \in E$.

Lemma 2.1: [13] If $p \in P$, then $\left|p_{k}\right| \leq 2(k=1,2,3, \ldots)$.

Lemma 2.2: [7,8] If $p \in P$, then

$$
\begin{gathered}
2 p_{2}=p_{1}^{2}+\left(4-p_{1}^{2}\right) x, \\
4 p_{3}=p_{1}^{3}+2 p_{1}\left(4-p_{1}^{2}\right) x-p_{1}\left(4-p_{1}^{2}\right) x^{2}+2\left(4-p_{1}^{2}\right)\left(1-|x|^{2}\right) z,
\end{gathered}
$$

For some $x$ and $z$ Satisfying $|x| \leq 1,|z| \leq 1$ and $p_{1} \in[0,2]$.

\section{MAIN RESULT}

Theorem 3.1: If $f \in M(\alpha ; A, B)$, then

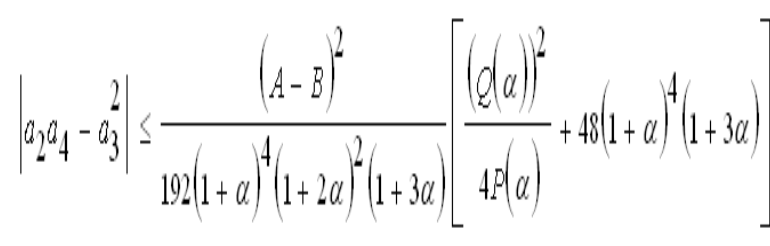

Where

$$
\begin{aligned}
& \left.P(\alpha)=-\mid \begin{array}{l}
\left\{\begin{array}{l}
6(1+5 \alpha)(1+3 \alpha)(1+2 \alpha)-4(1+2 \alpha)^{2}(1+7 \alpha)-3(1+3 \alpha)^{3} \\
6(1+5 \alpha)(1+\alpha)^{2}(1+2 \alpha)+6(1+5 \alpha)(1+3 \alpha)(1+2 \alpha) \\
+4(1+\alpha)^{3}(1+2 \alpha)^{2}-4(1+2 \alpha)^{2}(1+7 \alpha) \\
-3(1+3 \alpha)^{3}-3(1+\alpha)^{4}(1+3 \alpha)-6(1+3 \alpha)^{2}(1+\alpha)^{2}
\end{array}\right. \\
+\left\{B^{2}\right. \\
\left\{\begin{array}{l}
6(1+3 \alpha)^{3}+6(1+3 \alpha)^{2}(1+\alpha)^{2}-12(1+5 \alpha)(1+3 \alpha)(1+2 \alpha) \\
-6(1+5 \alpha)(1+\alpha)^{2}(1+2 \alpha)+8(1+2 \alpha)^{2}(1+7 \alpha)
\end{array}\right.
\end{array}\right\} A B \\
& +\left|6 \alpha(1+\alpha)^{3} A+2(1+\alpha)^{2}\left\{3(1+3 \alpha)\left(2+5 \alpha+\alpha^{2}\right)-(1+2 \alpha)\left(7+27 \alpha+8 \alpha^{2}\right)\right\} B\right| \\
& +(1+\alpha)^{3}\left\{1+4 \alpha+7 \alpha^{2}\right\} \\
& =1+B_{1} z+B_{2} z^{2}+B_{3} z^{3}+\ldots
\end{aligned}
$$

And

$$
\begin{aligned}
& Q(\alpha)=4 \begin{array}{l}
6 \alpha(1+\alpha)^{3} \mathrm{~A} \\
+2(1+\alpha)^{2}\left\{3(1+3 \alpha)\left(2+5 \alpha+\alpha^{2}\right)-(1+2 \alpha)\left(7+27 \alpha+8 \alpha^{2}\right)\right) B
\end{array} \mid \\
& -8(1+\alpha)^{3}\left[1+4 \alpha+\alpha^{2}\right]
\end{aligned}
$$

Proof: If $f(z) \in M(\alpha ; A, B)$, then there exists a Schwarz function $w(z) \in U$ such that

$$
(1-\alpha) \frac{z f^{\prime}(z)}{f(z)}+\alpha \frac{\left(z f^{\prime}(z)\right)^{\prime}}{f^{\prime}(z)}=\phi(w(z))
$$

Where

Define the function $p_{1}(z)$ by

$$
p_{1}(z)=\frac{1+w(z)}{1-w(z)}=1+c_{1} z+c_{2} z^{2}+c_{3} z^{3}+\ldots
$$

Since $w(z)$ is a Schwarz function, we see that $\operatorname{Re}\left(p_{1}(z)\right)>0$ and $p_{1}(0)=1$. Define the function $h(z)$ by

$$
h(z)=(1-\alpha) \frac{z f^{\prime}(z)}{f(z)}+\alpha \frac{\left(z f^{\prime}(z)\right)^{\prime}}{f^{\prime}(z)}=1+b_{1} z+b_{2} z^{2}+b_{3} z^{3}+.
$$

In view of the equations (3.4), (3.6) and (3.7), we have

$$
\phi(z)=\frac{1+A z}{1+B z}=1+(A-B) z-B(A-B) z^{2}+B^{2}(A-B) z^{3}+\ldots
$$




$$
\begin{aligned}
& h(z)=\phi\left(\frac{p_{1}(z)-1}{p_{1}(z)+1}\right)=\phi\left(\frac{c_{1} z+c_{2} z^{2}+c_{3} z^{3}+\ldots}{2+c_{1} z+c_{2} z^{2}+c_{3} z^{3}+\ldots}\right) \\
& b_{1}=\frac{B_{1} c_{1}}{2} ; b_{2}=\frac{B_{1}}{2}\left(c_{2}-\frac{c_{1}^{2}}{2}\right)+\frac{B_{2} c_{1}^{2}}{4} \\
& =\phi\left(\frac{1}{2} c_{1} z+\frac{1}{2}\left(c_{2}-\frac{c_{1}^{2}}{2}\right) z^{2}+\frac{1}{2}\left(c_{3}-c_{1} c_{2}+\frac{c_{1}^{3}}{4}\right) z^{3}+\ldots\right) \\
& =1+\frac{B_{1} c_{1}}{2} z+\left[\frac{B_{1}}{2}\left(c_{2}-\frac{c_{1}^{2}}{2}\right)+\frac{B_{2} c_{1}^{2}}{4}\right] z^{2} \\
& +\left[\frac{B_{1}}{2}\left(c_{3}-c_{1} c_{2}+\frac{c_{1}^{3}}{4}\right)+\frac{B_{2} c_{1}}{2}\left(c_{2}-\frac{c_{1}^{2}}{2}\right)+\frac{B_{3} c_{1}^{3}}{8}\right] z^{3}+\ldots
\end{aligned}
$$

Thus,

Using (3.5) and (3.7) in (3.8), we obtain

$$
\begin{aligned}
& a_{2}=\frac{(A-B) c_{1}}{2(1+\alpha)} \\
& a_{3}=\frac{(A-B)}{8(1+\alpha)^{2}(1+2 \alpha)}\left[\begin{array}{l}
2(1+\alpha)^{2} c_{2}+ \\
\left\{(1+3 \alpha) A-\left[(1+3 \alpha)+(1+\alpha)^{2}\right] B-(1+\alpha)^{2}\right\} c_{1}^{2}
\end{array}\right],
\end{aligned}
$$

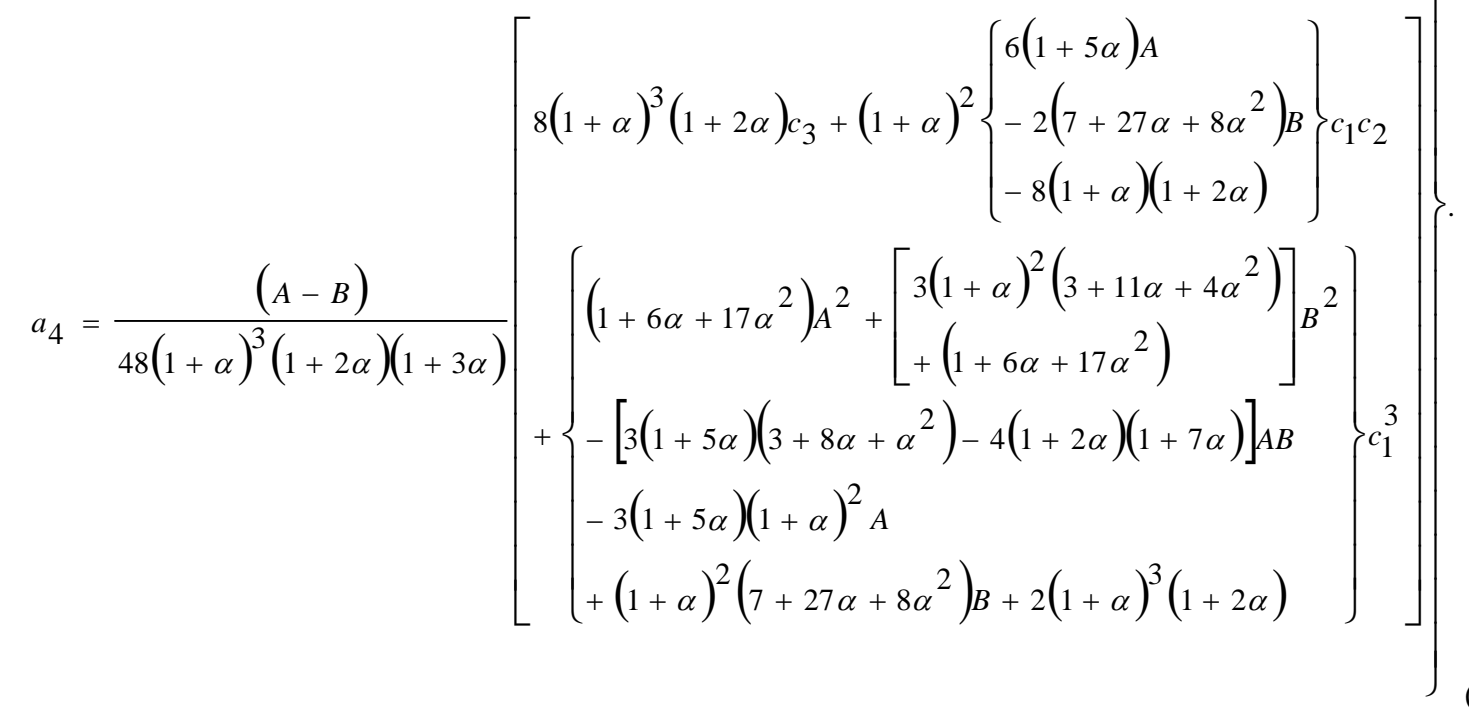

(3.9) yields,

$$
a_{2} a_{4}-a_{3}^{2}=\frac{(A-B)^{2}}{C(\alpha)}\left\{L c_{1}\left(4 c_{3}\right)+M c_{1}^{2}\left(2 c_{2}\right)+N c_{1}^{4}-R\left(4 c_{2}^{2}\right)\right\}
$$

Where

$$
\begin{gathered}
L=4(1+\alpha)^{3}(1+2 \alpha)^{2} \\
M=6 \alpha(1+\alpha)^{3} A+(1+\alpha)^{2}\left[\begin{array}{c}
12(1+3 \alpha)\left(2+5 \alpha+\alpha^{2}\right) \\
-4(1+2 \alpha)\left(7+27 \alpha+8 \alpha^{2}\right)
\end{array}\right] B \\
-2(1+\alpha)^{3}\left[1+4 \alpha+7 \alpha^{2}\right]
\end{gathered}
$$$$
C(\alpha)=192(1+\alpha)^{4}(1+2 \alpha)^{2}(1+3 \alpha),
$$ 


$$
\begin{aligned}
& N=\left[6(1+2 \alpha)(1+3 \alpha)(1+5 \alpha)-4(1+2 \alpha)^{2}(1+7 \alpha)-3(1+3 \alpha)^{3}\right] A^{2} \\
& +\left[\begin{array}{l}
6(1+2 \alpha)(1+5 \alpha)(1+\alpha)^{2}+6(1+2 \alpha)(1+3 \alpha)(1+5 \alpha) \\
+4(1+\alpha)^{3}(1+2 \alpha)^{2}-4(1+7 \alpha)(1+2 \alpha)^{2}-3(1+3 \alpha)^{3} \\
-3(1+\alpha)^{4}(1+3 \alpha)-6(1+3 \alpha)^{2}(1+\alpha)^{2}
\end{array}\right] B^{2} \\
& +\left[\begin{array}{l}
6(1+3 \alpha)^{3}+6(1+3 \alpha)^{2}(1+\alpha)^{2}-12(1+5 \alpha)(1+3 \alpha)(1+2 \alpha) \\
-6(1+5 \alpha)(1+\alpha)^{2}(1+2 \alpha)+8(1+2 \alpha)^{2}(1+7 \alpha)
\end{array}\right] A B \\
& -6 \alpha(1+\alpha)^{3} A+2(1+\alpha)^{2}\left[\begin{array}{l}
4(1+2 \alpha)^{2}(1+\alpha)+3(1+2 \alpha)(1+5 \alpha) \\
-3(1+3 \alpha)^{2}-3(1+\alpha)^{2}(1+3 \alpha)
\end{array}\right] B \\
& +(1+\alpha)^{3}\left[1+4 \alpha+7 \alpha^{2}\right]
\end{aligned}
$$

And

$$
R=3(1+3 \alpha)(1+\alpha)^{4} .
$$

Using Lemma 2.1 and Lemma 2.2 in (3.10), we get

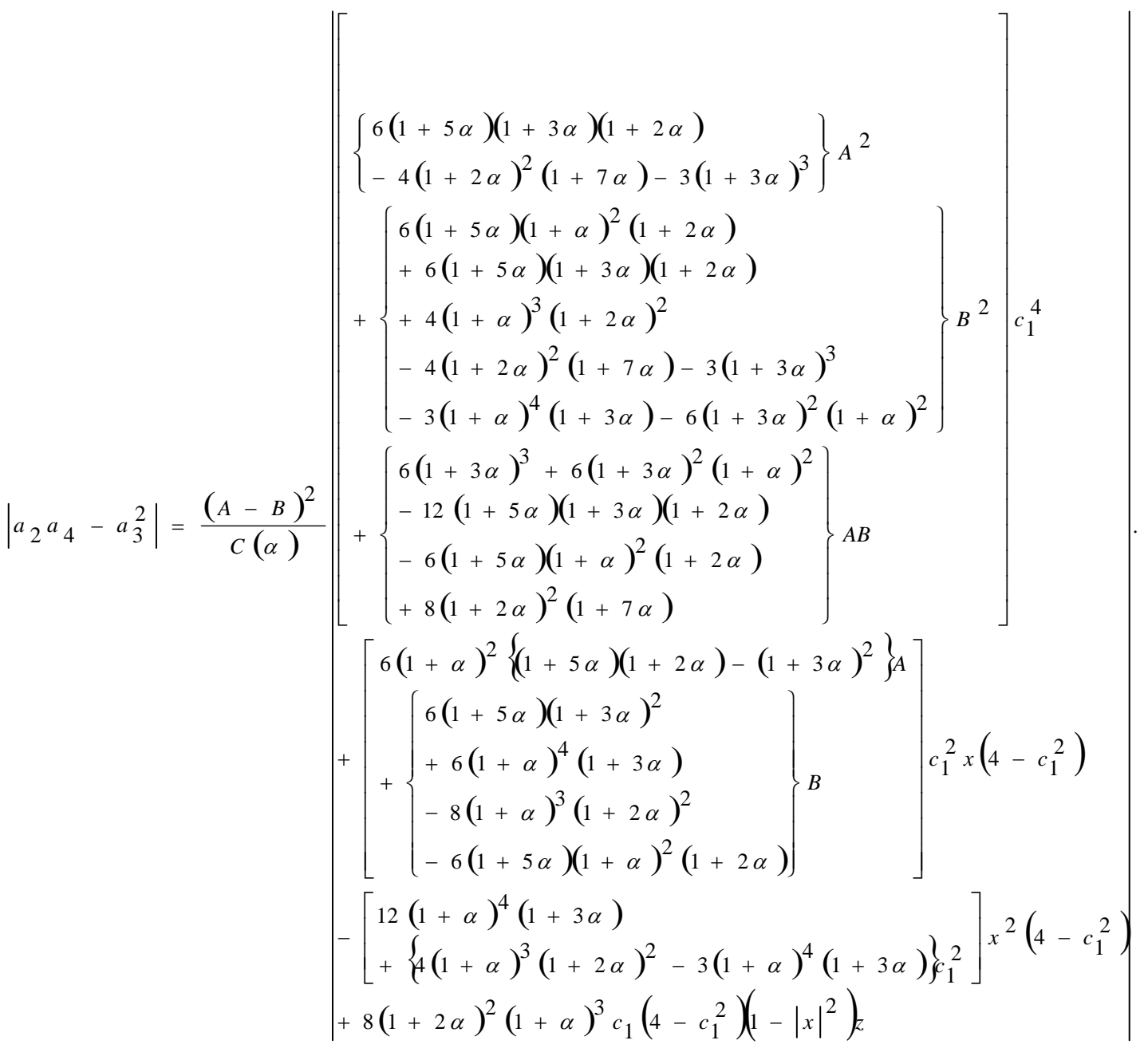


Assume that $c_{1}=c$ and $c \in[0,2]$, using triangular inequality and $|z| \leq 1$, we have

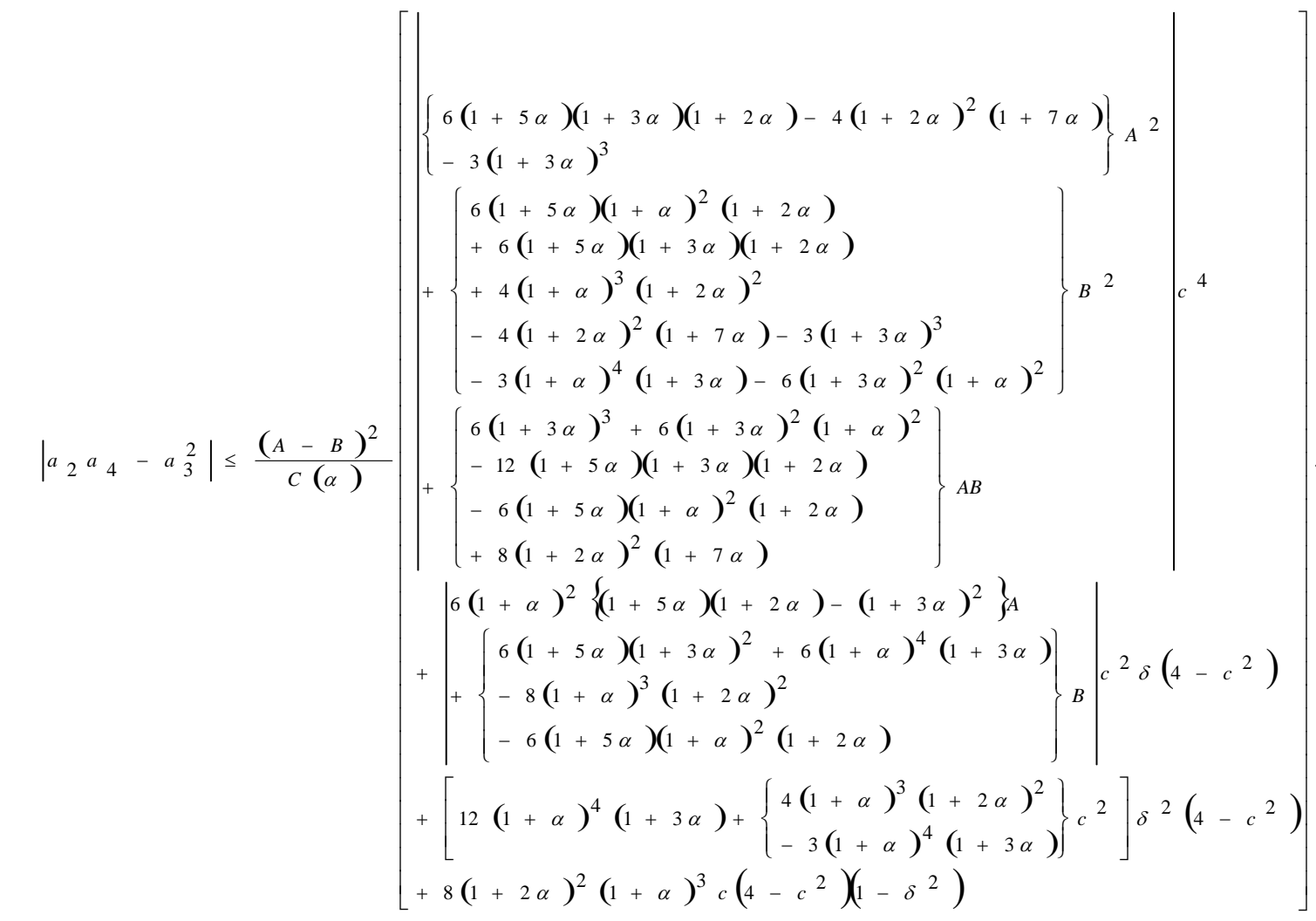

$=\frac{(A-B)^{2}}{C(\alpha)} F(\delta)$, where $\delta=|x| \leq 1$ and

$$
F(\delta)=\left\{\begin{array}{l}
\left\{\begin{array}{l}
6(1+5 \alpha)(1+3 \alpha)(1+2 \alpha) \\
-4(1+2 \alpha)^{2}(1+7 \alpha)-3(1+3 \alpha)^{3}
\end{array}\right\} A^{2} \\
+\left\{\begin{array}{l}
6(1+5 \alpha)(1+\alpha)^{2}(1+2 \alpha) \\
+6(1+5 \alpha)(1+3 \alpha)(1+2 \alpha)+4(1+\alpha)^{3}(1+2 \alpha)^{2} \\
-4(1+2 \alpha)^{2}(1+7 \alpha)-3(1+3 \alpha)^{3} \\
-3(1+\alpha)^{4}(1+3 \alpha)-6(1+3 \alpha)^{2}(1+\alpha)^{2}
\end{array}\right. \\
+\left\{\begin{array}{l}
6(1+3 \alpha)^{3}+6(1+3 \alpha)^{2}(1+\alpha)^{2} \\
-12(1+5 \alpha)(1+3 \alpha)(1+2 \alpha) \\
-6(1+5 \alpha)(1+\alpha)^{2}(1+2 \alpha)+8(1+2 \alpha)^{2}(1+7 \alpha)
\end{array}\right.
\end{array}\right\} B^{2}
$$

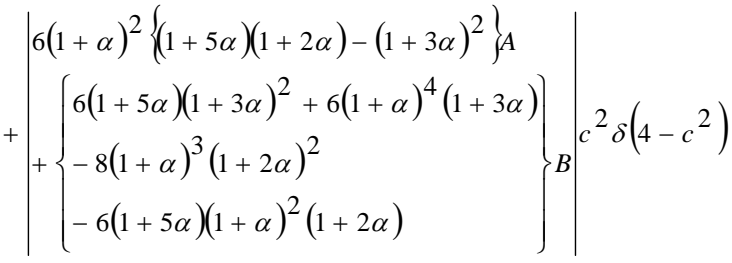$$
+\left[12(1+\alpha)^{4}(1+3 \alpha)+\left\{\begin{array}{c}
4(1+\alpha)^{3}(1+2 \alpha)^{2} \\
-3(1+\alpha)^{4}(1+3 \alpha)
\end{array}\right\} c^{2}\right] \delta^{2}\left(4-c^{2}\right)
$$$$
+8(1+2 \alpha)^{2}(1+\alpha)^{3} c\left(4-c^{2}\right)\left(1-\delta^{2}\right)
$$

Is an increasing function. Therefore $\operatorname{Ma} \boldsymbol{x}^{*}(\delta)=F(1)$.
Consequently

$$
\left|a_{2} a_{4}-a_{3}^{2}\right| \leq \frac{(A-B)^{2}}{C(\alpha)} G(c),
$$

Where

$$
G(c)=F(1)
$$

So

$$
G(c)=-P(\alpha) c^{4}+Q(\alpha) c^{2}+48(1+\alpha)^{4}(1+3 \alpha),
$$

Where $P(\alpha)$ and $Q(\alpha)$ are defined in (3.2) and (3.3) respectively.

Now

$$
G^{\prime}(c)=-4 P(\alpha) c^{3}+2 Q(\alpha) c
$$

And

$$
G^{\prime \prime}(c)=-12 P(\alpha) c^{2}+2 Q(\alpha) .
$$

$G^{\prime}(c)=0$ gives

$$
c\left\{-4 P(\alpha) c^{2}+2 Q(\alpha)\right\}=0 .
$$

$G^{\prime \prime}(c)$ is negative at $c=\sqrt{\frac{Q(\alpha)}{2 P(\alpha)}}=c^{\prime}$. 
So

$$
\operatorname{Max} G(c)=G\left(c^{\prime}\right)
$$

Hence from (3.11), we obtain (3.1).

The result is sharp for $c_{1}=c^{\prime}, \quad c_{2}=c_{1}^{2}-2$ and $c_{3}=c_{1}\left(c_{1}^{2}-3\right)$.

For $A=1$ and $B=-1$, Theorem 3.1 gives the following:

Corollary 3.1.1: If $f(z) \in M(\alpha)$, then

$$
\left|a_{2} a_{4}-a_{3}^{2}\right| \leq \frac{1}{(1+2 \alpha)^{2}}\left[1-\frac{12 \alpha^{2}(1+\alpha)^{4}}{(1+3 \alpha) A(\alpha)}\right],
$$

Where

$$
\begin{aligned}
& A(\alpha)=-24(1+2 \alpha)(1+5 \alpha)\left(2+5 \alpha+\alpha^{2}\right)+12(1+3 \alpha)^{2}\left(3+7 \alpha+2 \alpha^{2}\right) \\
& -4(1+\alpha)^{3}\left(1+4 \alpha+7 \alpha^{2}\right)+16(1+2 \alpha)^{2}(1+7 \alpha) .
\end{aligned}
$$

For $\alpha=0$, Theorem 3.1 gives the following result due to Singh and Singh [16].

Corollary 3.1.2: If $f(z) \in S^{*}(A, B)$, then

$$
\left|a_{2} a_{4}-a_{3}^{2}\right| \leq \frac{(A-B)^{2}}{4} \text {. }
$$

For $\alpha=1$, Theorem 3.1 gives the following result due to Singh and Singh [16].

Corollary 3.1.3: If $f \in K(A, B)$, then

$$
\left|a_{2} a_{4}-a_{3}^{2}\right| \leq \frac{(A-B)^{2}}{576}\left[\frac{16-A^{2}+2 B^{2}+A B|-| A-\left.5 B\right|^{2}-12|A-5 B|-36}{\left|-A^{2}+2 B^{2}+A B\right|-|A-5 B|-2}\right] .
$$

Putting $\alpha=0, A=1$ and $B=-1$ in Theorem 3.1, we obtain the following result due to Janteng et al. [5].

Corollary 3.1.4: If $f(z) \in S^{*}$, then

$$
\left|a_{2} a_{4}-a_{3}^{2}\right| \leq 1 \text {. }
$$

Putting $\alpha=1, A=1$ and $B=-1$ in Theorem 3.1, we obtain the following result due to Janteng et al. [5].

Corollary 3.1.5: If $f(z) \in K$, then

$$
\left|a_{2} a_{4}-a_{3}^{2}\right| \leq \frac{1}{8} \text {. }
$$

\section{REFERENCES}

[1] D. G. Cantor, Power series with integral coefficients, Bull. Amer. Math. Soc., Vol 69, pp 362-366, 1963.

[2] P. Dienes,The Taylor Series, Dover, New York, 1957.

[3] R. M. Goel and B. S. Mehrok,On the coefficients of a subclass of starlike functions, Ind. J. Pure and Appl. Math., vol.12, no. 5, pp 634$647,1981$.

[4] Aini Janteng, Suzeini Abdul Halim and Maslina Darus, Coefficient inequality for a function whose derivative has a positive real part, J. Ineq. Pure Appl. Math., vol.7, no.2, pp 1-5, 2006.
[5] Aini Janteng, Suzeini Abdul Halim and Maslina Darus,Hankel determinant for starlike and convex functions, Int. J. Math. Anal., vol.1, no.13, pp 619-625,2007.

[6] Aini Janteng, Suzeini Abdul Halim and Maslina Darus, Hankel determinant for functions starlike and convex with respect to symmetric points, J. Quality Measurement and Anal., vol.2, no.1,pp 3743, 2006.

[7] R. J. Libera and E-J. Zlotkiewiez, Early coefficients of the inverse of a regular convex function, Proc. Amer. Math. Soc., vol.85, pp225-230, 1982.

[8] R. J. Libera and E-J. Zlotkiewiez, Coefficient bounds for the inverse of a function with derivative in P, Proc. Amer. Math. Soc., vol.87, pp 251-257, 1983.

[9] T. H. MacGregor, Functions whose derivative has a positive real part, Trans. Amer. Math. Soc., vol.104, pp 532-537, 1962.

[10] B. S. Mehrok and Gagandeep Singh, Estimate of second Hankel determinant for certain classes of analytic functions, Scientia Magna, 8(3), 85-94, 2012.

[11] P. T. Mocanu, "Une propriété de convexité généralisée dans la théorie de la représentation conforme,” Mathematica, 11(34), 127-133, 1969.

[12] J. W. Noonan and D. K. Thomas, On the second Hankel determinant of a really mean p-valent functions, Trans. Amer. Math. Soc., vol. 223, no. 2, pp 337-346, 1976.

[13] Ch. Pommerenke, Univalent functions, Göttingen: Vandenhoeck and Ruprecht., 1975.

[14] Gagandeep Singh, Hankel determinant for new subclasses of analytic functions with respect to symmetric points, Int. J. of Modern Math. Sci., vol.5, no.2, pp 67-76, 2013.

[15] Gagandeep Singh, Hankel determinant for a new subclass of analytic functions, Scientia Magna, vol.8. no.4, pp 61-65, 2012.

[16] Gagandeep Singh and Gurcharanjit Singh, Second Hankel determinant for subclasses of starlike and convex functions, To Appear. 